\title{
EFEITO DA PARTICIPAÇÃO EM AULAS DE EDUCAÇÃO FÍSICA ES- COLAR SOBRE INDICADORES DE CRESCIMENTO, COMPOSIÇÃO CORPORAL E APTIDÃO NEUROMOTORA EM CRIANÇAS
}

\author{
Marcos Moura Santos \\ Universidade de Pernambuco, Recife, Pernambuco, Brasil \\ Daniel Rocha Queiroz \\ Universidade de Pernambuco, Recife, Pernambuco, Brasil \\ Teresinha de Jesus Sousa Lima \\ Universidade de Pernambuco, Recife, Pernambuco, Brasil \\ Mauricio Carneiro \\ Universidade de Pernambuco, Recife, Pernambuco, Brasil \\ Túlio Samuelson Martins Veloso \\ Universidade de Pernambuco, Recife, Pernambuco, Brasil \\ Victor Rafael de Lima Santos \\ Universidade de Pernambuco, Recife, Pernambuco, Brasil
}

\begin{abstract}
Resumo
O objetivo deste estudo foi examinar os efeitos de 12 semanas de aulas de Educação Física sobre os indicadores de crescimento, composição corporal e testes neuromotores em crianças. Foram avaliados 23 indivíduos, de ambos os sexos (6 a 10 anos), quanto às variáveis antropométricas (massa corporal total, estatura, altura sentada, IMC e RCQ), composição corporal e testes neuromotores (força, flexibilidade e RML abdominal). Para comparação das variáveis, a ANOVA two-way para medidas repetidas, seguida de Bonferroni, como post-hoc, e o teste $t$ pareado foram utilizados. Foram observadas alterações significativas positivas na massa corporal total, IMC, massa corporal magra e RML abdominal e uma redução significativa na RCQ $(\mathrm{p}<0,05)$. O envolvimento em aulas de Educação Física por um período de 12 semanas foi suficiente para promover alterações positivas em variáveis antropométricas, da composição corporal e neuromotoras em crianças de 6 a 10 anos de idade.
\end{abstract}

Palavras-chave: Atividade motora. Saúde do escolar. Crescimento. Aptidão física.

\section{Introdução}

Com a diminuição dos níveis de atividade física de crianças e adolescentes, a aptidão física relacionada à saúde tornou-se motivo de grande interesse para profissionais na área de saúde (TOMKINSON; OLDS, 2007; TOVAR et al., 2008). Neste contexto, alguns estudos têm discutido o real papel da Educação Física escolar no que concerne à prevenção e à pro- 
moção da saúde em populações pediátricas (GAYA et al., 2002; GLANER, 2003; FARIAS et al., 2010), preocupação válida, na medida que se considera a escola como um local primário para a prevenção e a promoção da saúde.

A preocupação com a melhoria na qualidade de vida e saúde da população tem se tornado uma questão de importância social, que incita a realização de estudos com o propósito de investigar a eficiência cardiorrespiratória e neuromuscular de crianças e adolescentes numa perspectiva de saúde e, consequentemente, permitindo ações intervencionistas (LUGUETTI; RE; BOHME, 2010; OLIVEIRA et al., 2017).

Ao considerarmos que a aptidão física é altamente sensível ao nível de atividade física, investigar o potencial efeito dos estímulos vivenciados em aulas de Educação Física, quando contrastados com estímulos ambientais positivos, é uma lacuna que necessita ser melhor esclarecida.

Neste contexto, a literatura tem dado especial enfoque ao acompanhamento do crescimento somático, da composição corporal e da aptidão física (SILVA; SILVA JUNIOR; OLIVEIRA, 2005), identificados como componentes morfológicos e funcionais que expressam, de alguma forma, os diferentes tipos de exposição aos quais crianças e adolescentes estão expostos, sejam eles nutricionais, sejam comportamentais. Os resultados reportados em estudos realizados com crianças e adolescentes (VERARDI et al., 2007; LUGUETTI; RE; BOHME, 2010; CARSON et al., 2016; POITRAS et al., 2016), apresentam melhores esclarecimentos sobre a variabilidade dos resultados observados nas diversas populações, o que permite estabelecer parâmetros mais consistentes (LUGUETTI; RE; BOHME, 2010).

Contudo, verifica-se a necessidade de um conhecimento sobre o quanto da participação em aulas de Educação Física escolar é suficiente para promover alterações em indicadores antropométricos, de composição corporal e testes neuromotores de crianças. Sendo assim, o objetivo deste estudo foi examinar os efeitos da participação durante 12 semanas em aulas de Educação Física Escolar sobre os parâmetros de crescimento somático, composição corporal e testes neuromotores em crianças dos 6 aos 10 anos de idade.

\section{Procedimentos metodológicos}

Este estudo caracteriza-se como do tipo pré-experimental de grupo único e faz parte do projeto de pesquisa intitulado "Crescimento somático, composição corporal, aptidão Física e coordenação motora: reflexos modulados pela condição ao nascer e a intervenção sistematizada". Todos os procedimentos foram aprovados pelo Comitê de Ética em Pesquisa com Seres Humanos da instituição de filiação dos autores e seguiu as normas estabelecidas pela Comissão Nacional de Ética e Pesquisa (CONEP), Resolução no 466/12, sobre pesquisa envolvendo seres humanos (CEP/UPE: 1.090.208). Um consentimento por escrito, assinado pelos pais ou responsável legal previamente ao início das coletas, e a própria aceitação das crianças foram requisitos para participação nesta pesquisa.

A amostra foi composta por 23 crianças (12 meninos), com idades entre 6 e 10 anos, matriculados em uma escola particular da cidade do Recife em turmas de $2^{\circ}$ ao $5^{\circ}$ ano do Ensino Fundamental I, escolhidas por conveniência e de forma intencional. Foram estabelecidos como critérios de inclusão dos participantes: a) estar devidamente matriculado na escola; b) apresentar autorização assinada pelos pais ou responsáveis legais. Como critério de exclusão: a) ter uma frequência abaixo de $80 \%$ nas 12 semanas propostas no estudo; b) não realizar em sua totalidade os testes e avaliações inicial e final do estudo. Nenhum dos voluntários submetidos às medidas e aos testes foi excluído do estudo. Cada indivíduo compareceu ao local de avaliação, acompanhado de um responsável para os seguintes procedimentos: 1) anamnese e esclarecimentos; 2) avaliação antropométrica; 3) avaliação neuromotora. 
Todos os procedimentos foram realizados na quadra poliesportiva da escola, durante as aulas de Educação Física. Foram realizadas as avaliações antropométricas e da aptidão neuromotora (momento 1; M1), em seguida foram registradas 12 semanas de participação nas aulas de Educação Física. Ao final deste período, os sujeitos foram reavaliados (momento 2; M2). Todos os protocolos utilizados para avaliação foram realizados por dois avaliadores devidamente treinados para este fim.

As aulas de Educação Física ministradas durante as 12 semanas foram estruturadas em um tempo máximo de 40 minutos, duas vezes por semana, totalizando 24 aulas. Os conteúdos foram tratados numa dimensão social, cultural, de saúde e motora, obedecendo ao Projeto Político Pedagógico da escola. Foram consideradas abordagens diferenciadas dos conteúdos entre as turmas.

Os escolares realizaram atividades consideradas habituais nas aulas de Educação Física. A dimensão de saúde teve um enfoque mais teórico, com aconselhamentos sobre alimentação saudável e higiene corporal e orientação sobre a importância da adoção de um estilo de vida ativo. A dimensão sociocultural abordou as questões históricas, éticas e culturais do esporte. A dimensão motora abordou o conteúdo esporte (futebol, futsal e voleibol) e jogos populares, utilizando, inicialmente, um modelo lúdico e recreativo como estratégia para estimular a participação. Os fundamentos dos esportes escolhidos foram abordados a partir da compreensão e do desenvolvimento das habilidades e capacidades motoras dos alunos de cada turma (MAGILL, 2011).

A avaliação dos parâmetros antropométricos das crianças incluiu: massa corporal total: obtida utilizando-se uma balança digital APA Speedo (Speedo, São Paulo, Brasil) com capacidade máxima de $150 \mathrm{Kg}$ e precisão de $100 \mathrm{~g}$. As crianças foram medidas com os trajes de Educação Física e descalças; estatura: foi obtida por meio de um estadiômetro Sanny ES2040 (Sanny, São Bernardo do Campo, Brasil), com precisão de 0,1 cm; índice de massa corporal (IMC): foi calculado pelo quociente entre a massa corporal total $(\mathrm{kg})$ e o quadrado da estatura (m); circunferências corporais (abdômen e cintura): foram obtidas em duplicata, por meio de uma trena metálica e inextensível (Cescorf, Porto Alegre, Brasil) com precisão de $0,1 \mathrm{~cm}$, segundo técnicas convencionais descritas previamente (CALLAWAY, 1991). O valor médio foi utilizado como referência. Com estas medidas, foi determinada a relação cintura/quadril (RCQ).

Para estimativa da composição corporal, foi utilizado o modelo de dois compartimentos (massa gorda e massa magra), adotando-se as medidas de espessuras de dobras cutâneas, por meio de um adipômetro científico (Lange, Santa Cruz, Califórnia) com pressão constante de $10 \mathrm{~g} / \mathrm{mm}^{2}$ e precisão de $1 \mathrm{~mm}$. Os pontos de coleta foram o triciptal e o subescapular e seguiram recomendações descritas previamente (SLAUGHTER et al., 1988). As medidas foram realizadas em triplicata e adotou-se como valor a mediana. A gordura corporal relativa foi estimada a partir do modelo matemático proposto por Slaughter et al. (1988).

Os dados da aptidão neuromotora foram coletados mediante a administração de uma bateria de testes motores compostos por quatro itens conhecidos e padronizados, obedecendo à seguinte sequência: flexibilidade (sentar e alcançar), força de membros superiores (medicine-ball), resistência abdominal (sit-ups) e força explosiva de membros inferiores (impulsão horizontal). Toda a avaliação da aptidão física foi efetuada com base nos protocolos da bateria de testes PROESP-BR (GAYA et al., 2012).

Foram realizados os testes de Shapiro Wilk e Levene, para testar a normalidade dos dados e a homogeneidade de variância, respectivamente. Por não atenderem aos pressupostos de normalidade, todas as variáveis sofreram transformação logarítmica. Inicialmente, foi testada a existência de interação entre os gêneros e os momentos (ANOVA two-way). Como não foram encontradas interações significativas, os grupos foram tratados em grupo único, isto é, meninos e meninas juntos. Inicialmente, o teste $t$ pareado foi utilizado para identificar as dife- 
renças entre os momentos nas características descritivas. Para comparações entre os momentos do estudo (basal e 12 semanas), utilizou-se a Análise de Variância de dois caminhos (ANOVA two-way), seguido da Bonferroni como post-hoc. Adicionalmente, foi calculado o tamanho do efeito (effect size) das intervenções, comparando-se os momentos (Cohen's $d$ ), sendo classificado mediante a proposição de Sawilowsky (2009). Os dados são expressos em média, desvio-padrão e os respectivos intervalos de confiança (IC95\%). O software Prism GraphPad (San Diego, Estados Unidos da América, versão 6.01) foi utilizado para a elaboração das figuras. Para a análise estatística, utilizou-se o software SPSS (SPSS Inc., Chicago, Estados Unidos da América, versão 10.0). Todas as análises utilizaram o teste bicaudal, estabelecendo o nível de significância em 5\%.

\section{Resultados}

A Tabela 1 apresenta as características descritivas da amostra entre os momentos inicial e final nas variáveis antropométricas. Foram observadas diferenças significativas na massa corporal total e no IMC após a participação regular em 12 semanas de aulas de Educação Física.

Tabela 1. Características descritivas da amostra e comparações entre os momentos 1 e 2 da intervenção em aulas de Educação Física

\begin{tabular}{lccccc}
\hline \multicolumn{1}{c}{ Variáveis } & \multicolumn{2}{c}{ Pré-intervenção $(\mathbf{n = 2 3})$} & Pós-intervenção $(\mathbf{n = 2 3})$ & p \\
\hline \multicolumn{1}{c}{ Antropometria } & Média $(\mathrm{dp})$ & IC95\% & Média $(\mathrm{dp})$ & IC95\% & \\
Massa Corporal total $(\mathrm{kg})$ & $32,01(10,54)$ & $27,46-36,57$ & $33,74(11,17)$ & $28,91-38,57$ & $<0,01$ \\
Estatura $(\mathrm{m})$ & $1,32(0,08)$ & $1,28-1,35$ & $1,33(0,10)$ & $1,29-1,37$ & 0,16 \\
IMC $\left(\mathrm{Kg} / \mathrm{m}^{2}\right)$ & $18,13(4,13)$ & $16,34-19,91$ & $18,77(4,32)$ & $16,90-20,64$ & $<0,05$ \\
\hline
\end{tabular}

* Utilizando o teste t pareado

Fonte: Os autores, 2016

A Tabela 2 apresenta os resultados das diferenças nas variáveis da composição corporal e neuromotoras. Foram identificadas diferenças estatisticamente significativas na massa corporal magra $(\mathrm{F}=38,79, \mathrm{p}<0,001 ; \mathrm{ES}=1,88$, efeito muito grande) e na $\mathrm{RML}$ abdominal $(\mathrm{F}=$ 9,25, $\mathrm{p}<0,01 ; \mathrm{ES}=0,92$, efeito grande). Destaca-se, que nestas variáveis, foram observadas alterações significativas condicionadas ao fator tempo $(\mathrm{p}<0,001)$. Porém, não se verifica diferença estatisticamente significativa quando observado o fator grupo (i.e., a interação não é significativa) ( $\mathrm{p}=0,16 ; \mathrm{p}=0,65$, respectivamente), demonstrando a efetividade do programa. $\mathrm{O}$ significado destes resultados é expresso pelo effect size observado. Já para o RCQ foram observadas diferenças significativas condicionadas pelo fator gênero $(\mathrm{p}<0,05)$. A interação é significativa RCQ $(F=7,78, p=0,011)$, demonstrando que as meninas foram mais responsivas e apresentaram um menor RCQ na pós-intervenção comparativamente aos meninos $(\mathrm{p}=0,047)$. As demais variáveis não apresentaram interação e diferenças significativas ( $>>0,05)$ 
Tabela 2. Comparação entre os diferentes momentos do estudo nas variáveis de composição corporal e testes neuromotores

\begin{tabular}{lcccccc}
\hline \multicolumn{1}{c}{ Variáveis } & Pré-intervenção & Pós-intervenção & \multicolumn{5}{c}{ Análise de Variância } \\
& $(\mathbf{n = 2 3 )}$ & $\mathbf{( n = 2 3 )}$ & $\mathbf{F}_{\text {Tempo }}$ & $\mathbf{p}$ & $\mathbf{F}_{\text {Gênero }}$ & $\mathbf{p}$ \\
\hline \multicolumn{1}{c}{ Composição Corporal } & & & & & & \\
$\sum$ TR+SE (mm) & $23,87(10,92)$ & $22,96(10,36)$ & 1,43 & 0,25 & 0,28 & 0,60 \\
RCQ & $0,82(0,06)$ & $0,81(0,06)$ & 0,31 & 0,58 & 7,46 & $<0,05$ \\
\% Gordura & $23,92(8,12)$ & $22,99(8,12)$ & 3,43 & 0,08 & 0,10 & 0,75 \\
Massa Gorda (kg) & $8,24(5,74)$ & $8,32(5,54)$ & 0,45 & 0,51 & 0,44 & 0,52 \\
Massa Corporal Magra (kg) & $23,78(5,63)$ & $25,43(6,26)$ & 38,79 & $<0,001$ & 2,17 & 0,16 \\
\hline \multicolumn{1}{c}{ Testes neuromotores } & & & & & & \\
Flexibilidade (cm) & $38,61(9,58)$ & $38,70(9,73)$ & 0,001 & 0,98 & 0,56 & 0,46 \\
RML abdômen (rep/min) & $21,74(10,14)$ & $26,04(11,19)$ & 9,25 & $<0,01$ & 0,21 & 0,65 \\
Força MS (metros) & $2,15(0,46)$ & $2,13(0,43)$ & 0,06 & 0,81 & 0,001 & 0,98 \\
Força de MI (metros) & $1,12(0,22)$ & $1,15(0,25)$ & 0,32 & 0,58 & 0,12 & 0,73 \\
\hline
\end{tabular}

Fonte: Os autores, 2016

A Figura 1 ilustra os resultados individuais das comparações apenas nas variáveis que apresentaram diferença entre o momento inicial e final.
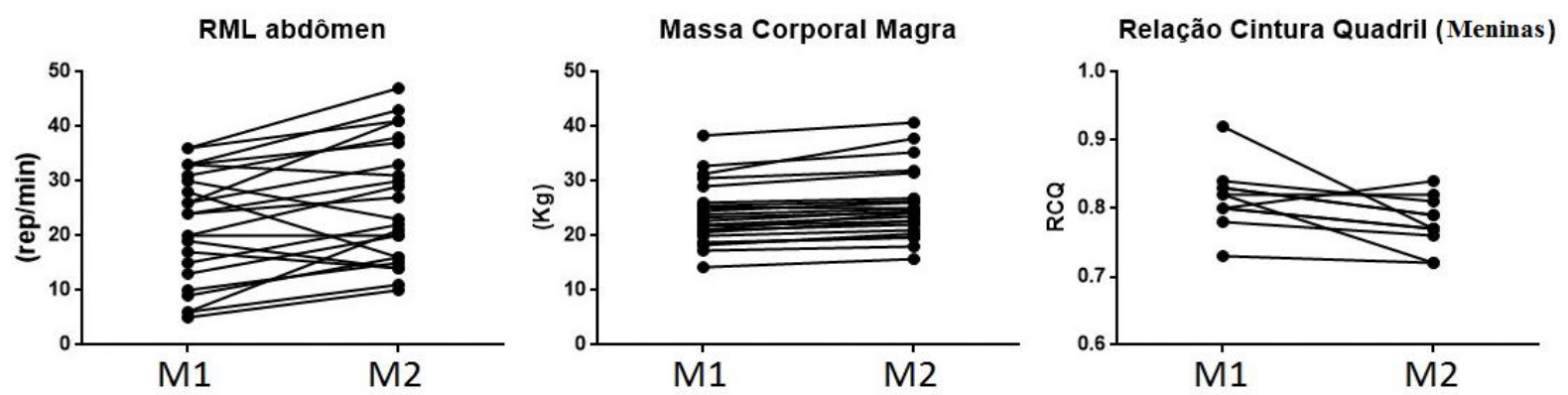

Figura 1. Resultados individuais da variação momento inicial (M1) vs momento final (M2)

Fonte: Os autores, 2016

\section{Discussão}

O objetivo do presente estudo foi examinar os efeitos da participação em aulas de Educação Física escolar nas variáveis de crescimento somático, composição corporal e testes neuromotores em crianças. Inicialmente, foi observado que, em relação às características de crescimento, composição corporal e testes neuromotores, as crianças se encontram dentro dos valores médios esperados para a idade e o gênero (DE ONIS, 2006; GAYA et al., 2012). Contudo, ao examinarmos estas variáveis após a realização de 12 semanas de aulas de Educação Física, foi verificado que os estímulos realizados promoveram alterações com comportamentos distintos, sejam refletidas em ganhos, sejam em reduções.

O envolvimento em práticas de atividades físicas é considerado como necessário, por exercer um papel modulador nos diferentes sistemas e tecidos ao longo das diferentes fases de 
crescimento e desenvolvimento (ROGOL; CLARK; ROEMMICH, 2000). De um ponto de vista educacional, as atividades no contexto escolar permitem diferentes experiências globais para crianças e jovens, tornando-se um importante caminho para a aprendizagem de novas habilidades, o envolvimento na prática de atividade física, interações sociais e a autocompreensão (MALINA, 2010).

Neste contexto, espera-se que o envolvimento nestas atividades promovam o desenvolvimento ativo e saudável (ROBINSON et al., 2015), de modo que possa induzir alterações em parâmetros relacionados à composição corporal, ao nível de atividade física e à aptidão física relacionada à saúde, variáveis que estão fortemente associadas ao aparecimento de doenças cardiovasculares, obesidade e taxas de mortalidade na população (TELAMA et al., 2005; NELSON et al., 2011; TREMBLAY et al., 2011; TIMPKA et al., 2014; SAUNDERS et al., 2016).

A participação nas aulas de Educação Física escolar possibilita um envolvimento com atividades organizadas que necessitam de uma sistematização adequada e um controle sobre a intensidade, a duração e a frequência dos diferentes estímulos propostos (PAFFENBARGER; LEE, 1996; KREMER et al., 2012). Um estudo de revisão sistemática avaliou as implicações decorrentes da prática de atividade física para a saúde em crianças e jovens, identificando a necessidade de uma prática com intensidade de moderada a vigorosa e com duração de 60 minutos/dia. Foi observado que esta intensidade e duração permitiram modificações em indicadores de saúde como a pressão arterial, a síndrome metabólica e a obesidade (JANSSEN; LEBLANC, 2010). Outro estudo de revisão sistemática avaliou a relação entre diferentes intensidades de atividade física (medidas objetivamente) com indicadores de saúde em crianças e adolescentes, identificando que a intensidade e a duração reportadas anteriormente trazem benefícios à saúde, além de ressaltar os potenciais benefícios de intensidades mais baixas e do tempo total da prática de atividade física para a prevenção de doenças e a promoção da saúde em escolares (POITRAS et al., 2016).

No presente estudo, as aulas de Educação Física escolar foram realizadas com uma frequência de duas vezes/semana e uma duração total de 80 minutos, representando uma parte das atividades físicas praticadas pelas crianças. Neste contexto, o acréscimo da frequência e da duração das aulas de Educação Física nem sempre é viável, dadas as exigências do currículo escolar, tornando necessário promover o envolvimento em atividades não formais de ensino e em estratégias para promover o uso eficiente do tempo de aula na Educação Física (DOBBINS et al., 2009), pois estas aulas geralmente não têm sua duração aproveitada por completo (KREMER et al., 2012).

No entanto, foi observado que a participação por 12 semanas nas aulas de Educação Física promoveu alterações na composição corporal e na aptidão física. É importante ressaltar que as alterações nessas variáveis muitas vezes não ocorrem de uma maneira mais efetiva em decorrência das adaptações metabólicas ocasionadas pela prática da atividade física e também através de mudanças no padrão de crescimento e de desenvolvimento provocadas pela própria passagem ao longo das diferentes idades (PARENT et al., 2003). Por exemplo, em um estudo realizado com crianças (Pernambuco - Brasil) de 7 a 10 anos de idade, agrupados de acordo com o peso ao nascer, foi observado que, após os ajustes para covariáveis (idade, gênero, composição corporal e nível de atividade física), não foram encontradas diferenças entre os sujeitos estudados (MOURA-DOS-SANTOS et al., 2013).

No presente estudo, foram observados uma diminuição na RCQ e um aumento na massa corporal magra e na RML abdominal. Soar, Vasconcelos \& Assis (2004), ao analisarem uma amostra de escolares (Santa Catarina - Brasil) de 7 a 9 anos, identificaram uma redução do RCQ ao longo dos anos entre as meninas e valores superiores no grupo de meninos. Nesse sentido, a diminuição desse índice em meninas parece refletir um fator que está associado ao início da entrada na puberdade, ao considerarmos o fato de as meninas serem mais 
precoces quanto a estes eventos, o que favorece o não surgimento de doenças cardiovasculares ao longo da vida. Destaca-se que o aumento proporcional nos valores de RCQ é reportado como um indicador de risco para essas doenças na fase adulta (POULIOT et al., 1994).

Relativamente ao aumento da massa corporal magra e à melhora no desempenho do teste de RML abdominal, estes podem indicar benefícios à saúde, ao considerarmos que níveis adequados de aptidão física (aptidão cardiorrespiratória, flexibilidade, força/resistência muscular e níveis adequados de gordura) estão relacionados ao menor risco de doenças crônico-degenerativas (GLANER, 2003; POETA et al., 2012). Por exemplo, Farias et al. (2010) analisaram a atividade física programada durante um ano letivo escolar em adolescentes (Rondônia - Brasil) de 10 a 15 anos em variáveis da aptidão física e não encontraram efeitos do tempo da intervenção sobre os escolares; porém, ao comparar os grupos (intervenção e controle), identificaram uma melhora nos testes de força/resistência em ambos os sexos. Em outro estudo, realizado por Poeta et al. (2012), foi analisada uma intervenção com escolares obesos (Santa Catarina - Brasil) de 8 a 11 anos. O grupo intervenção reduziu os indicadores de adiposidade e melhorou o desempenho no teste de força. Estes estudos identificaram um resultado inferior ou um decréscimo do desempenho nos grupos controle que não realizaram a atividade física programada. Outro estudo de revisão sistemática conduzida por Oliveira et al. (2017) teve como objetivo analisar o efeito de intervenções com modalidades esportivas coletivas sobre variáveis de aptidão física. Foi observada uma melhoria na composição corporal, resistência cardiorrespiratória e força de preensão manual em crianças com sobrepeso e obesidade em idade escolar; no entanto, não foram encontradas diferenças para variáveis de flexibilidade e de aptidão neuromotora.

É provável que as atividades propostas nas aulas de Educação Física escolar não estimulem em níveis adequados as capacidades motoras condicionais para o desenvolvimento de variáveis da aptidão física (força, resistência muscular, resistência cardiorrespiratória) (FARIAS et al., 2010). Dessa forma, os níveis de aptidão física e a composição corporal de crianças podem ser alterados por outros fatores. A atividade física habitual, fatores de ordem ambiental, nível socioeconômico, além das características da própria escola, como os espaços disponíveis para a prática, o conteúdo e a frequência das aulas de Educação Física, de alguma forma podem vir a influenciar na existência destas diferenças (MALINA; BOUCHAR; BAOR, 2004; ORTEGA et al., 2008; SANTOS et al., 2010; FARIAS et al., 2010).

Este estudo apresenta algumas limitações que devem ser consideradas. O controle da intensidade das atividades, o tempo total despendido por semana na prática de atividades físicas extracurriculares, o acompanhamento nutricional e a não utilização de grupo controle podem diminuir as possibilidades de generalização dos resultados. No entanto, as diferenças encontradas neste estudo apresentam tamanho de efeito grande ou superior, dado que a amostra foi reduzida e, mesmo assim, conseguiu identificar efeitos positivos das aulas de Educação Física nas variáveis de composição corporal e aptidão neuromotora.

\section{Conclusão}

Com base nos resultados encontrados, foi possível concluir que um programa de aulas de Educação Física Escolar (12 semanas) contribuiu para uma modificação no índice de massa corporal, massa corporal magra e na RML abdominal em crianças de 6 a 10 anos de idade. Além disto, houve uma redução na RCQ, e as meninas parecem ser mais responsivas a estes efeitos. Estes dados sugerem uma contribuição das aulas de Educação Física escolar na alteração de parâmetros antropométricos e da aptidão física. Recomenda-se a realização de estudos com um maior tempo de seguimento. 


\title{
EFFECT OF PARTICIPATION IN SCHOOL PHYSICAL EDUCATION CLASSES ON GROWTH INDICATORS, BODY COMPOSITION AND NEUROMOTOR FIT- NESS IN CHILDREN
}

\begin{abstract}
The aim of this study was to examine the effects of 12 weeks of physical education classes on somatic growth, body composition and neuromotor tests in children. Twenty-three (23) subjects of both sexes (6-10 years old) were evaluated for the anthropometric variables (weight, height, sitting height, BMI and WHR), body composition and neuromotor tests (muscle strength, flexibility and sit-up's). For comparison of variables, two-way ANOVA for repeated measures, followed by Bonferroni, as post-hoc and paired t-test were used. Significant positive results in total body mass, BMI, lean body mass and abdominal muscular endurance and a significant reduction in WHR $(\mathrm{p}<0,05)$ were observed.Participation in physical education classes for a period of 12 weeks was sufficient to promote positive changes in anthropometric variables, the body composition and neuromotor tests in children from 6 to 10 years.
\end{abstract}

Keywords: Motor activity. School health. Growth. Physical fitness.

\section{EFECTO DE LA PARTICIPACIÓN EN CLASES DE EDUCACIÓN FÍSICA ESCO- LAR SOBRE INDICADORES DE CRECIMIENTO, COMPOSICIÓN CORPORAL Y APTITUD NEUROMOTORA EM NIÑOS}

\section{Resumen}

El objetivo de este estudio fue examinar los efectos de 12 semanas de clases de Educación Física, tomando como base los indicadores de crecimiento, composición corporal y pruebas neuromotoras en niños. Se evaluaron 23 individuos, de ambos sexos (6 a 10 años), sobre las variables antropométricas (masa corporal total, estatura, altura sentada, IMC y RCQ), composición corporal y pruebas neuromotoras (fuerza, flexibilidad y RML abdominal). Para la comparación de las variables, la ANOVA two-way para medidas repetidas, seguido de Bonferroni, como post-hoc y la prueba $t$ pareada fueron utilizados. Se observaron alteraciones significativas positivas en la masa corporal total, IMC, masa corporal magra y RML abdominal y una reducción significativa en la RCQ $(\mathrm{p}<0,05)$. La participación en clases de Educación Física durante un período de 12 semanas fue suficiente para suscitar modificaciones positivas en variables antropométricas, de la composición corporal y neuromotoras en niños de 6 a 10 años de edad.

Palabras clave: Actividad motora. Salud escolar. Crecimiento. Aptitud física.

\section{Agradecimentos}

Agradecemos a todos os voluntários que se prontificaram em participar da pesquisa e à Profa Dra. Maria Emília Monteiro Higino da Silva Moura, da Secretaria de Educação - Governo do Estado de Pernambuco, por ter dado irrestrito apoio à realização desta pesquisa.

\section{Referências}

CALlAWAY, C. W. New weight guidelines for Americans. The American Journal of Clinical Nutrition, v. 54, n. 1, p. 171-172, 1991. 
CARSON, V. et al. Systematic review of sedentary behaviour and health indicators in schoolaged children and youth: an update. Applied Physiology, Nutrition, and Metabolism, v. 41, n. 6, p. S240-S265, 2016.

DE ONIS, M. Who child growth standards based on length/height, weight and age. Acta Paediatrica, v. 95, n. S450, p. 76-85, 2006.

DOBBINS, M.; HUSSON, H.; DeCORBY, K.; LaROCCA, R. L. School-based physical activity programs for promoting physical activity and fitness in children and adolescents aged 6 to 18. Cochrane Database of Systematic Reviews, 2009.

FARIAS, E. S.; CARVALHO, W. R. G.; GONÇALVES, E. M.; GUERRA-JUNIOR, G. Efeito da atividade física programada sobre a aptidão física em escolares adolescentes. Revista Brasileira de Cineantropometria e Desempenho Humano, v. 12, n. 2, p. 98-105, 2010.

GAYA, A. et al. Aptidão física relacionada à saúde: Um estudo piloto sobre o perfil de escolares de 7 a 17 anos da região sul do Brasil. Revista Perfil, v. 6, n. 6, p. 50-60, 2002.

GAYA, A.; LEMOS, A.; GAYA, A.; TEIXEIRA, D.; PINHEIRO, E.; MOREIRA, R. Projeto esporte Brasil (Proesp-Br): Manual de testes e avaliação. Porto Alegre: UFRGS/CNPQ/Ministério do esporte, 2012.

GLANER, M. F. Importância da atividade física relacionada à saúde. Revista Brasileira de Cineantropometria e Desempenho Humano, v. 5, n. 2, p. 75-85, 2003.

JANSSEN, I.; LEBLANC, A. G. Systematic review of the health benefits of physical activity and fitness in school-aged children and youth. International Journal of Behavioral Nutrition and Physical Activity, v. 7, n. 1, p. 1-16, 2010.

KREMER, M. M.; REICHERT, F. F.; HALLAL, P. C. Intensidade e duração dos esforços físicos em aulas de educação física. Revista de Saúde Pública, v. 46, n. 2, p. 320-26, 2012.

LUGUETTI, C. N.; RE, A. H. N.; BOHME, M. T. S. Indicadores de aptidão física de escolares da região centro-oeste da cidade de São Paulo. Revista Brasileira de Cineantropometria e Desempenho Humano, v. 12, n. 5, p. 331-37, 2010.

MAGILL, R. A. Aprendizagem e controle motor: conceitos e aplicações. São Paulo: Edgard Blucher, 2011.

MALINA, R. M. Physical activity and health of youth. Education, v. 10, n. 1, p. 21-42, 2010. MALINA, R. M.; BOUCHARD, C.; BA-OR, O. Growth, maturation, and physical activity. Champaign: Human Kinects, 2004.

MOURA-DOS-SANTOS, M. A.; BARROS, J.; ALMEIDA, M.; CASTRO, R.; MAIA, J.; LEANDRO, C. Permanent deficits in handgrip strength and running speed performance in low birth weight children. American Journal of Human Biology, v. 25, n. 1, p. 58-62, 2013.

NELSON, T. F.; STOVITZ, S. D.; THOMAS, M.; LAVOI, N. M.; BAUER, K. W.; NEUMARK-SZTAINER, D. Do youth sports prevent pediatric obesity? A systematic review and commentary. Current Sports Medicine Reports, v. 10, n. 6, p. 360-70, 2011. 
OLIVEIRA, A.; MONTEIRO, A.; JÁCOME, C.; AFREIXO, V.; MARQUES, A. Effects of group sports on health-related physical fitness of overweight youth: A systematic review and meta-analysis. Scandinavian Journal of Medicine \& Science in Sports, v. 27, n. 6, p. 604$11,2017$.

ORTEGA, F. B.; RUIZ, J. R.; CASTILlO, M. J.; SJOSTROM, M. Physical fitness in childhood and adolescence: A powerfull marker of health. International Journal of Obesity, $\mathrm{v}$. 32 , n. 1, p. 1-11, 2008

PAFFENBARGER, R. S.; LEE, M. Physical activity and fitness for health and longevity. Research Quarterly for Exercise and Sport, v. 67, n. S3, p. 11-28, 1996.

PARENT, A. S.; TEILMANN, G.; JULL, A.; SKAKKEBAEK, N. E.; TOPARRI, J.; BOURGUIGNON, J. P. The timing of normal puberty and the age limits of sexual precocity: Variations around the world, secular trends and changes after migrations. Endocrine Reviews, v. 24, n. 5, p. 668-93, 2003.

POETA, L. S.; DUARTE, M. F. S.; GIULIANO, I. C. B.; FARIAS JUNIOR, J. C. Intervenção interdisciplinar na composição corporal e em testes de aptidão física de crianças obesas. Revista Brasileira de Cineantropometria e Desempenho Humano, v. 14, n. 2, p. 134-43, 2012.

POITRAS, V. J. et al. Systematic review of the relationships between objectively measured physical activity and health indicators in school-aged children and youth. Applied Physiology, Nutrition, and Metabolism, v. 41, n. 6, p. S197-S239, 2016.

POULIOT, M. C. et al. Waist circumference and abdominal sagittal diameter: best simple anthropometric indexes of abdominal visceral adipose tissue accumulation and related cardiovascular risk in men and women. American Journal Cardiology, v. 73, n. 7, p. 460-468, 1994

ROBINSON, L. E.; STODDEN, D. F; BARNETT, L. M.; LOPES V.P.; LOGAN S.W.; RODRIGUES, L.P.; D'HONDT E. Motor competence and its effect on positive developmental trajectories of health. Sports Medicine, v. 45, n. 9, p. 1273-84, 2015.

ROGOL, A. D.; CLARK, P. A.; ROEMMICH, J. N. Growth and pubertal development in children and adolescents: Effects of diet and physical activity. The American Journal of Clinical Nutrition, v. 72, n. 2, p. 521s-528s, 2000.

SAUNDERS, T. J. et al. Combinations of physical activity, sedentary behaviour and sleep: relationships with health indicators in school-aged children and youth. Applied Physiology, Nutrition, and Metabolism, v. 41, n. 6, p. S283-293, 2016.

SAWILOWSKY, S. S. New effect size rules of thumb. Journal of Modern Applied Statistical Methods, v. 8, n. 2, p. 597-99, 2009.

SILVA, R. J. S.; SILVA JUNIOR, A. G.; OLIVEIRA, A. C. C. Crescimento em crianças e adolescentes: um estudo comparativo. Revista Brasileira de Cineantropometria e Desempenho Humano, v. 7, n. 1, p. 12-20, 2005. 
SANTOS, D. M. V.; CHAVES, R. N.; SOUZA, M. C.; SEABRA, A.; GARGANTA, R.; MAIA, J. A. R. Taxas de sucesso na aptidão física: efeitos da idade, sexo, atividade física, sobrepeso e obesidade. Revista Brasileira de Cineantropometria e Desempenho Humano, v. 12 , n. 5, p. 309-15, 2010.

SLAUGHTER, M. H. et al. Skinfold equations for estimation of body fatness in children and youth. Human Biology, v. 60, n. 1, p. 709-23, 1988.

SOAR, C.; VASCONCELOS, F. A. G.; ASSIS, M. A. A. A relação cintura quadril e o perímetro da cintura associados ao índice de massa corporal em estudo com escolares. Cadernos de Saúde Pública, v. 20, n. 6, p. 1609-16, 2004.

TELAMA, R.; YANG, X.; LAAKSO, L.; VIIKARI, J. Physical activity in childhood and adolescence as predictor of physical activity in young adulthood. American Journal of Preventive Medicine, v. 13, n. 4, p. 317-23, 2005.

TOMKINSON, G. R.; OLDS, T. S. Secular changes in aerobic fitness test performance of Australian children and adolescents. Basel (Switzerland): Karger Publishers, 2007.

TOVAR, G.; POVEDA, J. G.; PINILA, M. I.; LOBELO, F. Relationship between overweight, physical activity and physical fitness in school-aged boys in Bogotá Colombia. Arquivos Latino-americanos de Nutrición, v. 58, n. 3, p. 265-73, 2008.

TREMBLAY, M. S. et al. Systematic review of sedentary behavior and health indicators in school aged children. International Journal of Behavioral Nutrition and Physical Activity, v. 8, n. 1, p. 1-22, 2011.

TIMPKA, S.; PETERSSON, I. F.; ZHOU, C.; ENGLUND, M. Muscle strength in adolescent men and risk of cardiovascular disease events and mortality in middle age: A prospective cohort study. BMC Medicine, v. 12, n. 1, p. 62, 2014.

VERARDI, C. E. L.; LOBO, A. P. S.; AMARAL, V. E.; FREITAS, V. L.; HIROTA, V. B. Análise da aptidão física relacionada à saúde e ao desempenho motor em crianças e adolescentes da cidade de Carneirinho-MG. Revista Mackenzie de Educação Física e Esporte, v. 6, n. 3, p.127-134, 2009.

Recebido em: 05/10/2016

Revisado em: 11/03/2017

Aprovado em: 21/08/2017

Endereço para correspondência:

mmoura23@gmail.com

Marcos Moura Santos

Universidade de Pernambuco, Conselho de Ensino, Pesquisa e Extensão.

Rua Anorbio Marques, 310

Santo Amaro

50100130 - Recife, PE - Brasil 